\title{
SU(4) flavor symmetry breaking in D-meson couplings to light hadrons
}

\author{
C.E. Fontoura ${ }^{1,2}$, J. Haidenbauer ${ }^{3}$, and G. Krein ${ }^{2, \text { a }}$ \\ 1 Instituto Tecnológico da Aeronáutica, DCTA, 12228-900 São José dos Campos, SP, Brazil \\ 2 Instituto de Física Teórica, Universidade Estadual Paulista, 01140-070 São Paulo, SP, Brazil \\ 3 Institute for Advanced Simulation, Institut für Kernphysik, and Jülich Center for Hadron Physics, Forschungszentrum Jülich, \\ D-52425 Jülich, Germany
}

Received: 3 February 2017 / Revised: 10 April 2017

Published online: 16 May 2017

(c) The Author(s) 2017. This article is published with open access at Springerlink.com

Communicated by L. Tolos

\begin{abstract}
The validity of $S U(4)$-flavor symmetry relations of couplings of charmed $D$-mesons to light mesons and baryons is examined with the use of ${ }^{3} P_{0}$ quark-pair creation model and nonrelativistic quarkmodel wave functions. We focus on the three-meson couplings $\pi \pi \rho, K K \rho$ and $D D \rho$ and baryon-baryonmeson couplings $N N \pi, N \Lambda K$ and $N \Lambda_{c} D$. It is found that $S U(4)$-flavor symmetry is broken at the level of $30 \%$ in the $D D \rho$ tree-meson couplings and $20 \%$ in the baryon-baryon-meson couplings. Consequences of these findings for $D N$ cross sections and existence of bound states $D$-mesons in nuclei are discussed.
\end{abstract}

\section{Introduction}

Currently there is considerable interest in exploring the interactions of charmed hadrons with light hadrons and atomic nuclei [1]. Particular attention is paid to $D$-mesons, much discussed over the last few years in connection with $D$-mesic nuclei $[2-4]$ and $J / \psi$ binding to nuclei $[5,6]$. Presently, there is no experimental information about the $D N$ interaction, a situation that the $\overline{\mathrm{P}} \mathrm{ANDA} @ \mathrm{FAIR}$ experiment [7] could remedy in the future. Most of the knowledge on the $D N$ interaction comes from calculations using hadronic Lagrangians motivated by $S U(4)$ extensions of light-flavor chiral Lagrangians [8-14] and heavy quark symmetry $[15,16]$. These require as input coupling constants and, in some cases, form factors. For the particular case of $\bar{D} N$ reactions (where $\bar{D} \equiv\left\{\bar{D}^{0}, D^{-}\right\}$), ref. [11] found that among all the couplings in the effective Lagrangian, $g_{D D \rho}$ and $g_{D D \omega}$ provide the largest contributions to cross sections and phase shifts for kinetic center of mass (c.m.) energies up to $150 \mathrm{MeV}$ - they also play an important role for the $D N$ interaction [13]. Flavor $S U(4)$ symmetry relates those couplings to couplings in the lightflavor sector,

$$
\begin{gathered}
g_{D D \rho}=g_{K K \rho}=\frac{1}{2} g_{\pi \pi \rho}, \\
g_{N \Lambda_{c} D}=g_{N \Lambda K}=\frac{3 \sqrt{3}}{5} g_{N N \pi} .
\end{gathered}
$$

\footnotetext{
${ }^{a}$ e-mail: gkrein@ift.unesp.br
}

The studies in refs. [11-13] utilized the $S U(4)$ relations above, based on $g_{\pi \pi \rho}=6.0$ and $g_{N N \pi}=13.6$, which are the values used in a large body of work conducted within the Jülich model $[17,18]$ for light-flavor hadrons.

Given the prominent role played by meson-baryon Lagrangians in the study of the $D N$ interaction and associated phenomena, it is of utmost importance to assess the validity of (1) and (2). $S U(4)$ breaking effects on three-hadron couplings were examined recently using a variety of approaches, that include vector meson dominance (VMS) [19, 20], Dyson-Schwinger and BetheSalpeter equations (DS-BS) of QCD [21], QCD sum rules (QCDSR) [22-24], lattice QCD [25], and holographic QCD [26]. In this work we use the quark model with a ${ }^{3} P_{0}$ quark-pair creation operator [27-29]. In this setting, the three-hadron couplings are given by matrix elements of the ${ }^{3} P_{0}$ operator evaluated with quark-model wave functions. The literature on the ${ }^{3} P_{0}$ model is too vast to be properly reviewed here, we simply mention that it is being used extensively since the early 1970s to study strong decays and that our calculation of vertices shares similarities with those of nucleon-meson couplings and form factors in [27-30].

\section{Three-hadron couplings}

To evaluate the matrix element of the ${ }^{3} P_{0}$ quark-pair creation operator, $\hat{O}_{\mathrm{pc}}$, it is convenient to employ the "decay frame" of an initial hadron at rest $[27-30]$, i.e. the 
transition of a hadron state $\left|h_{1}\right\rangle$ into a final two-hadron state $\left|h_{2} h_{3}\right\rangle$ is written as

$$
\left\langle h_{2} h_{3}\left|\hat{O}_{\mathrm{pc}}\right| h_{1}\right\rangle \equiv \delta\left(\boldsymbol{P}_{1}-\boldsymbol{P}_{2}-\boldsymbol{P}_{3}\right) \mathcal{M}_{h_{1} h_{2} h_{3}}(\boldsymbol{q}),
$$

with $\boldsymbol{q}=\boldsymbol{P}_{2}=-\boldsymbol{P}_{3}$, and

$$
\hat{O}_{\mathrm{pc}}=\gamma \sum_{c f s s^{\prime}} \int \mathrm{d}^{3} p \boldsymbol{\sigma}_{s^{\prime} s}^{c} \cdot \boldsymbol{p} q_{s^{\prime}}^{c f \dagger}(\boldsymbol{p}) \bar{q}_{s}^{c f \dagger}(-\boldsymbol{p}),
$$

where $\gamma$ gives the strength of the quark-pair creation, $q_{s^{\prime}}^{c f^{\dagger}}(\boldsymbol{p})$ and $\bar{q}_{s}^{c f \dagger}(\boldsymbol{p})$ are creation operators with color $c$, flavor $f$, spin projection $s$, and momentum $\boldsymbol{p}, \boldsymbol{\sigma}_{s^{\prime} s}^{c}=\chi_{s^{\prime}}^{\dagger} \boldsymbol{\sigma} \chi_{s}^{c}$, with $\boldsymbol{\sigma}=\left(\sigma^{1}, \sigma^{2}, \sigma^{3}\right)$ being the Pauli matrices, $\chi_{s}$ Pauli spinors, and $\chi_{s}^{c}=-i \sigma^{2} \chi_{s}^{*}$. [31],

We employ the standard quark-model Hamiltonian

$$
\begin{aligned}
H= & \sum_{i}\left(m_{i}+\frac{p_{i}^{2}}{2 m_{i}}\right)-\sum_{i<j} \boldsymbol{F}_{i} \cdot \boldsymbol{F}_{j}\left[\left(\frac{3}{4} b r_{i j}-\frac{\alpha_{c}}{r_{i j}}\right)\right. \\
& \left.+\frac{8 \pi \alpha_{s}}{3 m_{i} m_{j}}\left(\frac{\sigma^{3}}{\pi^{\frac{3}{2}}} e^{-\sigma^{2} r_{i j}^{2}}\right) \boldsymbol{S}_{i} \cdot \boldsymbol{S}_{j}\right]
\end{aligned}
$$

where $m_{i}$ are the quark masses and $\boldsymbol{F}=\boldsymbol{\lambda} / 2$, with $\boldsymbol{\lambda}$ the color $S U(3)$ Gell-Mann matrices and $\boldsymbol{S}$ the spin $1 / 2$ vector. Notwithstanding the inability of the model to describe all features associated with the Goldstone-boson nature of the pion, nonetheless it mimics some of the effects of dynamical chiral symmetry breaking, notably the $\pi-\rho$ mass splitting [32]. As in QCD itself, the only source of $S U(4)$ breaking in (5) is the quark-mass matrix and hence the breaking in the couplings comes solely from the hadron wave functions. The Schrödinger equation is solved as a generalized matrix problem using a finite basis of Gaussian functions with the eigenvalues determined by the Rayleigh-Ritz variational principle. Reasonable values for the masses of the ground states of the hadrons of interest can be obtained by expanding the meson $\Phi$ and baryon $\Psi$ intrinsic wave functions as $[31,33]$

$$
\Phi(\boldsymbol{r})=\sum_{n=1}^{N} c_{n} \varphi_{n}(\boldsymbol{r}), \quad \Psi(\boldsymbol{\rho}, \boldsymbol{\lambda})=\sum_{n=1}^{N} c_{n} \varphi_{n}(\boldsymbol{\rho}) \varphi_{n}(\boldsymbol{\lambda})
$$

where the $c_{n}$ are dimensionless expansion parameters and

$$
\varphi_{n}(\boldsymbol{x})=\left(\frac{n \alpha^{2}}{\pi}\right)^{3 / 4} e^{-n \alpha^{2} \boldsymbol{x}^{2} / 2}
$$

Here, $\alpha$ is the variational, $\boldsymbol{r}=\boldsymbol{r}_{1}-\boldsymbol{r}_{2}, \boldsymbol{\rho}=\left(\boldsymbol{r}_{1}-\boldsymbol{r}_{2}\right) / \sqrt{2}$, and $\boldsymbol{\lambda}=\sqrt{2 / 3}\left[\left(\boldsymbol{r}_{1}+\boldsymbol{r}_{2}\right) / 2-\boldsymbol{r}_{3}\right]$. The matrix element $\mathcal{M}(\boldsymbol{q})$ can be evaluated analytically; it is given by

$$
\mathcal{M}_{h_{1} h_{2} h_{3}}(\boldsymbol{q})=\kappa_{h_{1} h_{2} h_{3}} A_{h_{1} h_{2} h_{3}}(\boldsymbol{q})|\boldsymbol{q}| Y_{1 m}(\hat{\boldsymbol{q}}),
$$

where $Y_{1 m}(\hat{\boldsymbol{q}})$ are spherical harmonics with $m=1(0)$ for three-meson (nucleon-baryon-meson) couplings, $\kappa$ comes from summing over color, spin, and flavor and is given by

$$
\begin{aligned}
\kappa_{D D \rho} & =\kappa_{K K \rho}=\frac{1}{2} \kappa_{\pi \pi \rho}=1, \\
\kappa_{N \Lambda_{c} D} & =\kappa_{N \Lambda K}=\frac{3 \sqrt{3}}{5} \kappa_{N N \pi}=1 .
\end{aligned}
$$

The amplitude $A_{h_{1} h_{2} h_{3}}(\boldsymbol{q})$ in (8) is given by

$$
\begin{aligned}
A_{h_{1} h_{2} h_{3}}(\boldsymbol{q})= & \gamma \sum_{n_{1} n_{2} n_{3}} c_{n_{3}}^{*} c_{n_{2}}^{*} c_{n_{1}}\left(n_{1} n_{2} n_{3}\right)^{3 / 4} \\
& \times f_{h_{1} h_{2} h_{3}}\left(n_{1}, n_{2}, n_{3}\right) e^{-\boldsymbol{q}^{2} / \Lambda_{h_{1} h_{2} h_{3}}^{2}\left(n_{1}, n_{2}, n_{3}\right)},
\end{aligned}
$$

where $f_{h_{1} h_{2} h_{3}}$ are given by ( $P$ in $P P \rho$ stands for $\pi, K, D$ and $B$ in $N B P$ for $N, \Lambda, \Lambda_{c}$ )

$$
\begin{aligned}
& f_{P P \rho}\left(n_{1}, n_{2}, n_{3}\right)=\left(\frac{64}{9 \pi}\right)^{1 / 4} \frac{\alpha_{\rho}^{3 / 2}}{\alpha_{P}^{3}} \\
& \times \frac{n_{1} n_{2}+\left(\bar{m}_{1} n_{1} n_{3}+2 n_{2} n_{3}\right) \alpha_{\rho}^{2} / \alpha_{P}^{2}}{\left[n_{1} n_{2}+\left(n_{1}+n_{2}\right) n_{3} \alpha_{\rho}^{2} / \alpha_{P}^{2}\right]^{5 / 2}} \\
& f_{N B P}\left(n_{1}, n_{2}, n_{3}\right)=\frac{72}{\pi^{3 / 4}} \frac{\alpha_{B}^{3}}{\alpha_{N}^{3} \alpha_{P}^{3 / 2}} \frac{1}{\left(n_{1}+n_{2} \alpha_{B}^{2} / \alpha_{N}^{2}\right)^{3 / 2}} \\
& \times \frac{\bar{m}_{2} n_{1} n_{2} \alpha_{B}^{2} / \alpha_{P}^{2}+\widetilde{m}_{2} n_{1} n_{3}+3 n_{2} n_{3} \alpha_{B}^{2} / \alpha_{N}^{2}}{\left(2 n_{1} n_{2} \alpha_{B}^{2} / \alpha_{P}^{2}+3 n_{1} n_{3}+3 n_{2} n_{3} \alpha_{B}^{2} / \alpha_{N}^{2}\right)^{5 / 2}}
\end{aligned}
$$

and the "cut-off" parameters $\Lambda_{h_{1} h_{2} h_{3}}$ are given by

$$
\begin{aligned}
& \Lambda_{P P \rho}^{2}\left(n_{1}, n_{2}, n_{3}\right)=\frac{8 \alpha_{P}^{2}\left[n_{1} n_{2}+\left(n_{1}+n_{2}\right) n_{3} \alpha_{\rho}^{2} / \alpha_{P}^{2}\right]}{(\Delta \bar{m})^{2} n_{1}+n_{2}+n_{3} \bar{m}_{2}^{2} \alpha_{\rho}^{2} / \alpha_{P}^{2}}, \\
& \Lambda_{N B P}^{2}\left(n_{1}, n_{2}, n_{3}\right)=\frac{24 \alpha_{P}^{2}}{\bar{m}_{1}^{2}} \\
& \times \frac{2 n_{1} n_{2} \alpha_{B}^{2} / \alpha_{P}^{2}+3 n_{1} n_{3}+3 n_{2} n_{3} \alpha_{B}^{2} / \alpha_{N}^{2}}{n_{1} \widetilde{m}_{2}^{2}+9 n_{2} \alpha_{B}^{2} / \alpha_{N}^{2}+6\left(\widetilde{m}_{1}+\widetilde{m}_{2}\right)^{2} n_{3} \alpha_{P}^{2} / \alpha_{N}^{2}},
\end{aligned}
$$

where

$$
\bar{m}_{1,2}=\frac{2 m_{1,2}}{m_{2}+m_{1}}, \quad \widetilde{m}_{1,2}=\frac{3 m_{1,2}}{2 m_{1}+m_{2}}, \quad \Delta \bar{m}=\frac{m_{2}-m_{1}}{m_{2}+m_{1}},
$$

with $m_{1}=m_{u}=m_{d}, m_{2}=m_{s}, m_{c}$.

In the limit of $S U(4)$ symmetry, $m_{1}=m_{2}, \alpha_{D}=\alpha_{K}=$ $\alpha_{\pi}$ and $\alpha_{\Lambda_{c}}=\alpha_{\Lambda}=\alpha_{N}$, and the ratios

$$
\mathcal{R}_{P / P^{\prime}}\left(\boldsymbol{q}^{2}\right)=\frac{A_{P P \rho}\left(\boldsymbol{q}^{2}\right)}{A_{P^{\prime} P^{\prime} \rho}\left(\boldsymbol{q}^{2}\right)}, \quad \mathcal{R}_{B P / B^{\prime} P^{\prime}}\left(\boldsymbol{q}^{2}\right)=\frac{A_{N B P}\left(\boldsymbol{q}^{2}\right)}{A_{N B^{\prime} P^{\prime}}\left(\boldsymbol{q}^{2}\right)}
$$

are all equal to 1 , expressing the same symmetry as in (1) and (2). In this limit, $\gamma$ must be the same for all couplings, which seems a reasonable assumption, as they involve the same light-quark pair creation. Symmetry-breaking effects are contained in the factors $f, c_{n}$ and $\Lambda$.

Let us now connect to meson-exchange models. A typical three-meson vertex function, as it appears in that approach in the $P N$ potentials (with $P=K, \bar{K}, \bar{D}, D)[11-$ $13]$, is given by (in the decay frame)

$$
A_{P P V}\left(\boldsymbol{q}^{2}\right)=\phi_{\mathrm{KF}} g_{P P V}\left(\frac{\Lambda_{P P V}^{2}-m_{V}^{2}}{\Lambda_{P P V}^{2}-q^{2}}\right)^{n}|\boldsymbol{q}| Y_{11}(\hat{\boldsymbol{q}}) .
$$

Here $\phi_{\mathrm{KF}}$ is a kinematical factor involving the energies of the hadrons, $g_{P P V}$ is the coupling constant in the Lagrangian, and there is also a form factor with a cutoff 
Table 1. Calculated hadron masses $\left(m_{\text {calc }}\right)$ and sizes $(\alpha)$. Experimental values for the masses $\left(m_{\exp }\right)$ are from the PDG [34]. All values are in $\mathrm{MeV}$.

\begin{tabular}{ccccc|ccc}
\hline & $\pi$ & $K$ & $D$ & $\rho$ & $N$ & $\Lambda$ & $\Lambda_{c}$ \\
\hline$m_{\text {calc }}$ & 138 & 495 & 1866 & 770 & 958 & 1115 & 2195 \\
$m_{\exp }$ & 138 & 495 & 1866 & 770 & 940 & 1115 & 2286 \\
\hline$\alpha$ & 359 & 377 & 499 & 275 & 234 & 241 & 253 \\
\hline
\end{tabular}

mass $\Lambda_{P P V}$, where $n=1$ or $n=2[17,18]$. Here, the value of $g_{P P V}$ refers to the case when the vector meson $V$ is on its mass shell. Then $q^{2}=\left(q^{0}\right)^{2}-\boldsymbol{q}^{2}=m_{V}^{2}$ and the form factor is 1 . For low-energy elastic $P N$ scattering, the exchanged $\rho$ (and $\omega$ ) meson is far from its mass shell; the momentum transfer $q^{2}$ is small and negative, i.e. $q^{2}=\left(q^{0}\right)^{2}-\boldsymbol{q}^{2} \equiv-\boldsymbol{q}^{2}$ with $\boldsymbol{q}^{2} \gtrsim 0$. Therefore, it is common practice to use the static approximation $q^{2}=-\boldsymbol{q}^{2}$ in the form factors. We note that for the $D N(\bar{D} N)$ processes studied in refs. [11-14] up to kinetic c.m. energy of $150 \mathrm{MeV}$, the highest c.m. momentum is $400 \mathrm{MeV} / c$. The cutoff mass in the form factors is another source of symmetry breaking in the meson-exchange potentials. However, in the $D N(\bar{D} N)$ interactions in $[11-13]$ those masses were simply taken over from the corresponding $\bar{K} N(K N)$ interactions, for $\rho$ as well as for $\omega$ exchange. Thus, they drop out in the ratio (17).

The situation with baryon exchange is much more complicated, as different baryons are exchanged in the $\bar{D} N$ and $D N$ reactions. The separation of kinematical effects and the coupling strength, as in (18), cannot be easily done. Indeed in $\bar{D} N(K N)$ elastic scattering only $B=\Lambda_{c}$ $(\Lambda)$ exchange contributes while for $D N(\bar{K} N)$ there is only $N$ exchange, and only in the transitions $D N \leftrightarrow \pi \Lambda_{c}$ $(\bar{K} N \leftrightarrow \pi \Lambda)$. Furthermore, for heavy baryons like $\Lambda_{c}$ an extrapolation to the pole is rather questionable as the quark-model is not expected to work at such high momenta. Despite these drawbacks, we include here our baryon results for illustration purposes.

\section{Results}

We use the quark-model parameters of [31]: $m_{l}=$ $375 \mathrm{MeV}, m_{s}=650 \mathrm{MeV}, \alpha_{c}=0.857, \alpha_{s}=0.84, b=$ $0.154 \mathrm{GeV}^{2}, \sigma=70 \mathrm{MeV}$. We take $m_{c}=1657 \mathrm{MeV}$ to fit the $D$-meson mass. Table 1 shows the results; convergence is achieved with $N=11$ Gaussian functions. Clearly, the model fits well the experimental values of the masses, the largest discrepancy is $4 \%$ in the mass of $\Lambda_{c}$. In particular, the $\rho-\pi$ and $N-\Lambda$ mass splittings are well described. In addition, $m_{\Sigma}-m_{\Lambda}=82 \mathrm{MeV}$ and $m_{\Sigma_{c}}-m_{\Lambda_{c}}=135 \mathrm{MeV}$, also in fair agreement with data [34]. Since the corresponding effects on the $\Sigma$ and $\Sigma_{c}$ wave functions have a very small effect on the coupling constants, we consider only those couplings involving $\Lambda$ and $\Lambda_{c}$. We take $m_{u}=m_{d}$ so that $m_{\rho}=m_{\omega}$.

The ratios $\mathcal{R}\left(\boldsymbol{q}^{2}\right)$ are shown in fig. 1; we recall, $P P \rho$ couplings enter graphs with $\rho$ exchange and $N B P$ couplings in graphs with baryon $B$ exchanges. Figure 1 reveals
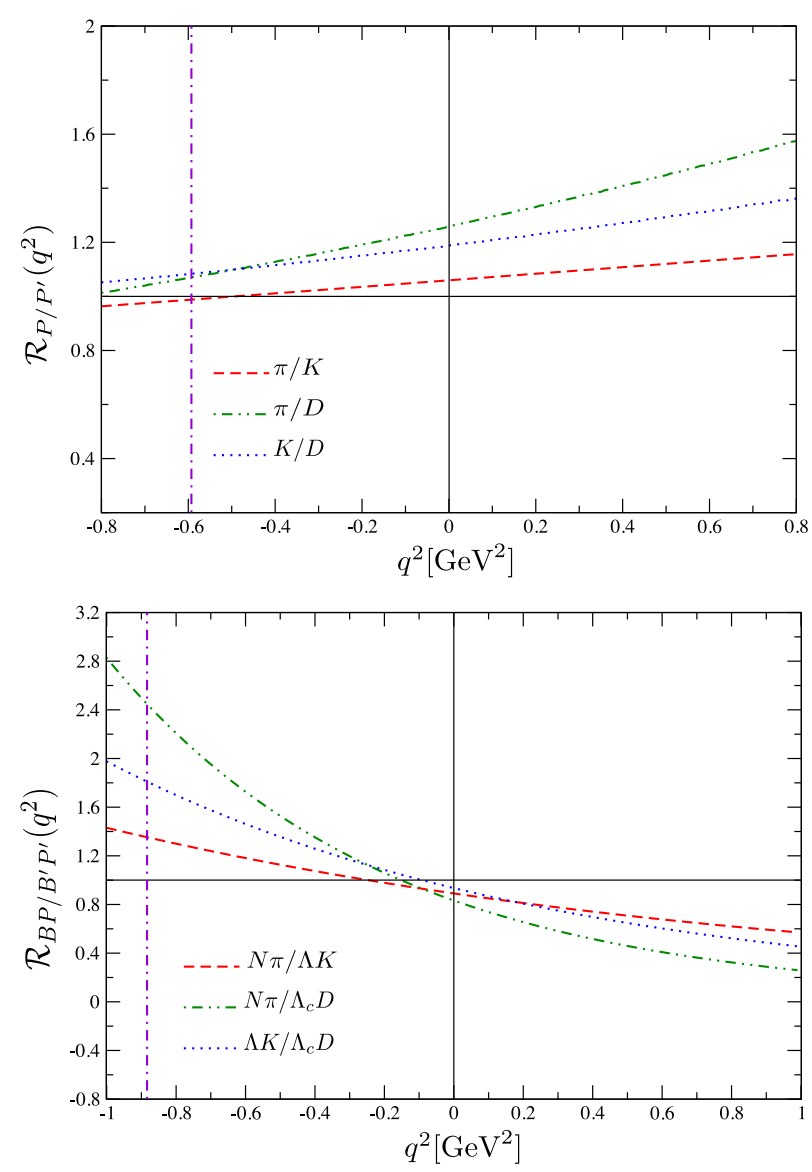

Fig. 1. Ratios $\mathcal{R}_{P / P^{\prime}}$ and $\mathcal{R}_{B P / B^{\prime} P^{\prime}}$; the vertical lines correspond to $\boldsymbol{q}^{2}=-m_{\rho}^{2}$ (top) and $\boldsymbol{q}^{2}=-m_{N}^{2}$ (bottom).

that $S U(4)$ breaking, at $\boldsymbol{q}^{2}=0$ and $\boldsymbol{q}^{2}=-m_{\rho}^{2}$, is relatively modest. At $\boldsymbol{q}^{2}=0$, the largest $S U(4)$ breaking, not unexpectedly, is in $D D \rho$, of the order of $30 \%$ compared to $\pi \pi \rho$ coupling, and $20 \%$ compared to $K K \rho$. Moreover, in agreement with phenomenology, there is almost no $S U(3)$ breaking in $K K \rho$. At the $\rho$ pole $\left(\boldsymbol{q}^{2}=-m_{\rho}^{2}\right)$ the breaking is also small, at most $10 \%$ in $D D \rho$ coupling. The ratios of $N B P$ couplings are presented in the bottom panel of the figure. As can be seen, the $S U(4)$ breaking at $\boldsymbol{q}^{2}=0$ is at most $20 \%$ in the $N \Lambda_{c} D$ vertex compared to the $N N \pi$ coupling and $10 \%$ compared to the $N \Lambda K$. The $S U(3)$ symmetry breaking in the $N \Lambda K$ coupling is of the order of $10 \%$, i.e. also compatible with phenomenology. Interestingly, for $\boldsymbol{q}^{2} \approx-0.9 \mathrm{GeV} / c$, i.e. close to the nucleon pole (for orientation, shown by the vertical line in the bottom panel of fig. 1), the $N \Lambda_{c} D$ coupling is 3 times smaller than the $N N \pi$ coupling, while the ratio of the $N \Lambda K$ to $N \Lambda_{c} D$ couplings is around 1.8. This is to be compared with the value 0.68 in [23]. However, such possible $S U(4)$ breaking far into the time-like region might not be relevant for low-energy $\bar{D} N$ scattering because, according to [11], the contribution of $\Lambda_{c}$ exchange to the $\bar{D} N$ cross section is very small anyway.

Physically, the $S U(4)$ breaking originates from the different extensions of the hadron wave functions. In fig. 2, 


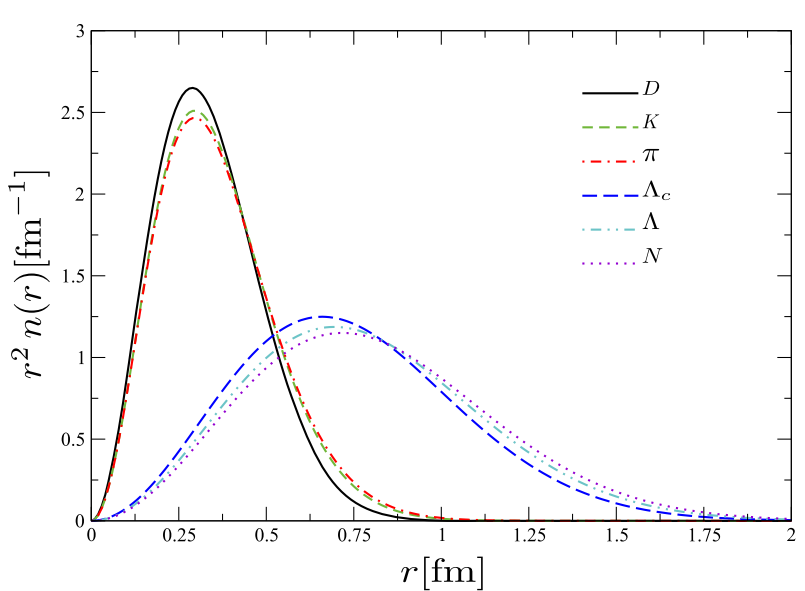

Fig. 2. Normalized light-quark radial distributions in mesons and baryons.

Table 2. Ratios of three-hadron couplings. In case of exact $S U$ (4) symmetry all ratios would be equal to 1 (see text).

\begin{tabular}{cccc}
\hline$P / P^{\prime}$ & $\pi / K$ & $\pi / D$ & $K / D$ \\
${ }^{3} P_{0} \mathcal{R}(0)$ & 1.05 & 1.26 & 1.19 \\
${ }^{3} P_{0} \mathcal{R}\left(-m_{\rho}^{2}\right)$ & 0.99 & 1.07 & 1.08 \\
Ref. [21] & 1.09 & 0.21 & 0.19 \\
Ref. [26] & 1.11 & 2.23 & 2.00 \\
\hline$B P / B^{\prime} P^{\prime}$ & $N \pi / \Lambda K$ & $N \pi / \Lambda_{c} D$ & $\Lambda K / \Lambda_{c} D$ \\
${ }^{3} P_{0} \mathcal{R}(0)$ & 0.89 & 0.83 & 0.92 \\
Ref. $[23]$ & - & - & 0.68 \\
\hline
\end{tabular}

we plotted the normalized light-quark radial distribution functions in the hadrons of interest - the Fourier transform of $\left\langle h\left|q^{\dagger}(\boldsymbol{q}) q(\boldsymbol{q})\right| h\right\rangle$. The distributions get more compact (shorter-ranged) for heavier hadrons as the binding increases due to smaller kinetic energies of the heavy quarks. This implies a smaller $P$ - $\rho$ overlap and thereby a smaller coupling. For $N B P$ fig. 2 shows that the $B$ $P$ overlap increases because the large- $r$ part of the light quark distribution in $B$ is cut off by the one from $P$, which explains the increased values of the couplings for heavier baryons. Figure 2 makes the physics transparent and explains the modest effects on the couplings.

We have also computed the coupling constants $g_{P P \rho}$ and $g_{N B P}$ of the Lagrangians in [11] by matching the ${ }^{3} P_{0}$ transition amplitude $\mathcal{M}_{h_{1} h_{2} h_{3}}$ in (3) to the one calculated with those Lagrangians. The matching is done at tree level at $\boldsymbol{q}^{2}=0$ [27-30]. Taking the typical values for $\gamma$ of the literature, $\gamma=0.4-0.5$ [30], the matching leads to $g_{\pi \pi \rho}=5.85-7.32$ and $g_{N N \pi}=10.83-13.54$, that are in very good agreement with phenomenology, and $g_{K K \rho}=2.79-3.49, g_{D D i \rho}=2.34-2.90, g_{N \Lambda K}=$ 12.65-15.81, $g_{N \Lambda_{c} D}=13.56-16.95$. In table 2 we collected the ratios of these couplings and quoted results from the literature. The ratios include isospin factors, as in (1) and (2) - for exact $S U(4)$ symmetry, the ratios are 1 . The value for $g_{D D i \rho}$ agrees well with $\operatorname{VMD}[19,20]$, QCDSR [22], and lattice QCD [25], and agrees within a factor of 2 with DS-BS [21] and holographic QCD [26].

\section{Summary}

We used a ${ }^{3} P_{0}$ quark-pair creation model with nonrelativistic quark-model wave functions to investigate the effects of $S U(4)$ symmetry breaking in the $D D \rho$ and $N \Lambda_{c} D$ couplings, the most relevant ones for the $\bar{D} N$ and $D N$ interactions $[11,13]$. The quark masses in the Hamiltonian (5) are the only source of $S U(4)$ breaking. The predictions of the model are reliable for lowmomentum transfers in the vertices. The pattern found for $S U(4)$ breaking for momenta $\boldsymbol{q}^{2} \approx 0$ in the $P P \rho$ amplitudes is $A_{D D \rho}<A_{K K \rho}<A_{\pi \pi \rho}$, while for $N B P$ it is $A_{N \Lambda_{c} D}>A_{N \Lambda K}>A_{N N \pi}$. Since the $D D \rho($ and $D D \omega)$ coupling is more important for the $\bar{D} N$ cross section than the $N \Lambda_{c} D$ (and $N \Sigma_{c} D$ ) coupling, at least in the calculations in [11-14], our results indicate that the use of $S U(4)$ symmetry for the coupling constants could be a reasonable first approximation, in line with other studies in the literature $[19,20,23,25,26]$. Clearly, for estimating the impact of our findings for the $S U(4)$ breaking on $D N$ cross sections, and also binding energies of $D$-mesic nuclei, further detailed studies are required. Finally, we note that the symmetry breaking pattern we found for $P P \rho$ couplings is opposite to that in ref. [21], but it agrees with the one in the holographic QCD calculation in [26]. We found also an opposite ratio for $N \Lambda K / N \Lambda_{c} D$ to the one in [23]. Further studies are needed for full clarification.

The work of GK was supported by CNPq Grant No. 305894/2009-9 and FAPESP Grant No. 2013/01907-0 and CEF was funded by CNPq Grant No. 150659/2015-6.

Open Access This is an open access article distributed under the terms of the Creative Commons Attribution License (http://creativecommons.org/licenses/by/4.0), which permits unrestricted use, distribution, and reproduction in any medium, provided the original work is properly cited.

\section{References}

1. R.A. Briceño et al., Chin. Phys. C 40, 042001 (2016).

2. K. Tsushima et al., Phys. Rev. C 59, 2824 (1999).

3. C. Garcia-Recio et al., Phys. Lett. B 690, 369 (2010).

4. C. Garcia-Recio et al., Phys. Rev. C 85, 025203 (2012).

5. S.H. Lee, C.M. Ko, Phys. Rev. C 67, 038202 (2003).

6. G. Krein et al., Phys. Lett. B 697, 136 (2011).

7. U. Wiedner, Prog. Part. Nucl. Phys. 66, 477 (2011).

8. T. Mizutani, A. Ramos, Phys. Rev. C 74, 065201 (2006).

9. Z.-w. Lin et al., Phys. Rev. C 61, 024904 (2000).

10. J. Hofmann, M.F.F. Lutz, Nucl. Phys. A 763, 90 (2005).

11. J. Haidenbauer et al., Eur. Phys. J. A 33, 107 (2007).

12. J. Haidenbauer et al., Eur. Phys. J. A 37, 55 (2008).

13. J. Haidenbauer et al., Eur. Phys. J. A 47, 18 (2011).

14. C.E. Fontoura et al., Phys. Rev. C 87, 025206 (2013).

15. S. Yasui, K. Sudoh, Phys. Rev. D 80, 034008 (2009).

16. C. Garcia-Recio et al., Phys. Rev. D 79, 054004 (2009).

17. J. Haidenbauer et al., Phys. Rev. C 45, 931 (1992).

18. M. Hoffmann et al., Nucl. Phys. A 593, 341 (1995).

19. S. Matinyan, B. Müller, Phys. Rev. C 58, 2994 (1998). 
20. Z.w. Lin, C.M. Ko, Phys. Rev. C 62, 034903 (2000).

21. B. El-Bennich et al., Phys. Rev. D 85, 031502 (2012).

22. M.E. Bracco et al., Prog. Part. Nucl. Phys. 67, 1019 (2012).

23. A. Khodjamirian et al., Eur. Phys. J. A 48, 31 (2012).

24. F.S. Navarra, M. Nielsen, Phys. Lett. B 443, 285 (1998).

25. K.U. Can et al., Phys. Lett. B 719, 103 (2013).

26. A. Ballon-Bayona et al., arXiv:1702.08417 [hep-ph].

27. L. Micu, Nucl. Phys. B 10, 521 (1969).
28. A. Le Yaouanc et al., Phys. Rev. D 8, 2223 (1973)

29. A. Le Yaouanc et al., Phys. Rev. D 9, 1415 (1974).

30. C. Downum et al., Phys. Lett. B 638, 455 (2006).

31. E.S. Swanson, Ann. Phys. 220, 73 (1992).

32. A.P. Szczepaniak, E.S. Swanson, Phys. Rev. Lett. 87, 072001 (2001).

33. B. Silvestre-Brac et al., Nucl. Phys. A 589, 585 (1995).

34. K.A. Olive et al., Chin. Phys. C 38, 090001 (2014). 